\title{
Copy-number variation is an important contributor to the genetic causality of inherited retinal degenerations
}

\author{
Kinga M. Bujakowska', Rosario Fernandez-Godino ${ }^{1}$, Emily Place ${ }^{1}$, Mark Consugar ${ }^{1}$, \\ Daniel Navarro-Gomez ${ }^{1}$, Joseph White', Emma C. Bedoukian², Xiaosong Zhu², Hongbo M. Xie ${ }^{3}$, \\ Xiaowu Gai ${ }^{4}$, Bart P. Leroy ${ }^{2,5}$ and Eric A. Pierce ${ }^{1}$
}

\begin{abstract}
Purpose: Despite substantial progress in sequencing, current strategies can genetically solve only approximately $55-60 \%$ of inherited retinal degeneration (IRD) cases. This can be partially attributed to elusive mutations in the known IRD genes, which are not easily identified by the targeted next-generation sequencing (NGS) or Sanger sequencing approaches. We hypothesized that copy-number variations (CNVs) are a major contributor to the elusive genetic causality of IRDs.
\end{abstract}

Methods: Twenty-eight cases previously unsolved with a targeted NGS were investigated with whole-genome single-nucleotide polymorphism (SNP) and comparative genomic hybridization (CGH) arrays.

Results: Deletions in the IRD genes were detected in 5 of 28 families, including a de novo deletion. We suggest that the de novo deletion occurred through nonallelic homologous recombination (NAHR)

\section{INTRODUCTION}

Inherited retinal degenerations (IRDs) are important causes of blindness that affect more than 2 million people worldwide. ${ }^{1}$ They are a family of blinding diseases characterized by progressive death and dysfunction of rod and cone photoreceptor cells. ${ }^{1,2}$ There are several major clinical subtypes of IRDs, with the most common being retinitis pigmentosa (RP), which is also called rod-cone dystrophy and accounts for approximately $25 \%$ of vision loss in adults. ${ }^{2}$ Other subtypes of IRDs have been reported, such as those predominantly affecting cones and/or the macula and pan-retinal degenerations such a Leber congenital amaurosis. ${ }^{1}$ Retinal degeneration is also one of the clinical manifestations of syndromic disorders such as Usher syndrome and Bardet-Biedl syndrome. ${ }^{1,2}$ Even though distinct IRD subtypes have been identified, there is considerable phenotypic overlap between the different types of IRD, which becomes even more apparent upon genetic testing. ${ }^{1}$ and we constructed a genomic map of NAHR-prone regions with overlapping IRD genes. In this article, we also report an unusual case of recessive retinitis pigmentosa due to compound heterozygous mutations in SNRNP200, a gene that is typically associated with the dominant form of this disease.

Conclusions: CNV mapping substantially increased the genetic diagnostic rate of IRDs, detecting genetic causality in $18 \%$ of previously unsolved cases. Extending the search to other structural variations will probably demonstrate an even higher contribution to genetic causality of IRDs.

Genet Med advance online publication 13 October 2016

Key Words: cone dystrophy; copy-number variation; Jalili syndrome; retinitis pigmentosa; rod-cone dystrophy

\footnotetext{
${ }^{1}$ Ocular Genomics Institute, Massachusetts Eye and Ear Infirmary, Department of Ophthalmology, Harvard Medical School, Boston, Massachusetts, USA; ${ }^{2}$ Ophthalmic Genetics \& Visual Electrophysiology, Division of Ophthalmology, The Children's Hospital of Philadelphia, Philadelphia, Pennsylvania, USA; ${ }^{3}$ Department of BioMedical Health Informatics, Children's Hospital of Philadelphia, Philadelphia, Pennsylvania, USA; ${ }^{4}$ Center for Personalized Medicine, Children's Hospital Los Angeles, Los Angeles, California, USA; ${ }^{5}$ Department of Ophthalmology \& Center for Medical Genetics, Ghent University Hospital \& Ghent University, Ghent, Belgium. Correspondence: Eric A. Pierce (eric_pierce@meei.harvard.edu) 


\section{ORIGINAL RESEARCH ARTICLE}

investigated CNVs in 28 genetically unsolved families using a dense single-nucleotide polymorphism (SNP) array (Illumina Human Omni 2.5/5.0) and/or a genome-wide comparative genomic hybridization (CGH) array. We detected large deletions in known IRD disease genes in five families, thereby demonstrating that a combined approach of targeted NGS and deletion mapping can lead to an estimated diagnostic rate of $67 \%$ of IRD cases. In two families, we detected essentially the same 1.1-Mb deletion spanning two IRD genes (SNRNP200 and CNNM4). The recurrent deletion probably occurred through the mechanism of nonallelic homologous recombination (NAHR), in which the rearranged genomic regions are flanked by paralogous repeat sequences. ${ }^{12}$ We have therefore created a genome-wide map of NAHR-prone regions and superimposed known IRD genes. Overall, 35 IRD genes and four genes with deletions reported in this study (SNRNP200, CNNM4, OPN1LW, and OPN1MW) matched these regions.

\section{Patient cohort}

\section{MATERIALS AND METHODS}

The study protocol adhered to the tenets of the Declaration of Helsinki and was approved by the institutional review boards of Massachusetts Eye and Ear Infirmary (MEEI, Harvard Medical School) and the Children's Hospital of Philadelphia (CHOP, University of Pennsylvania Perelman School of Medicine). The patients included in the study were recruited and clinically examined at MEEI and/or CHOP. Patients underwent a full ophthalmic examination that included best-corrected Snellen visual acuity, dynamic Goldmann visual field testing with the I-4e and V-4e objects of Goldmann, dark adaptation testing performed after 45 minutes of dark adaptation with an 11-degree white test light in the Goldmann-Weekers dark adaptometer, full-field electroretinographic (ERG) testing with assessment of $0.5-\mathrm{Hz}$ ERG amplitude and $30-\mathrm{Hz}$ ERG amplitudes (MEEI), and ISCEV standard ERG testing (CHOP). After patients signed informed consent, blood samples were collected from patients and available family members for DNA extraction and genetic analysis.

\section{Selective exon capture and WES}

For custom selective exon capture, paired-end SureSelect targeted enrichment capture libraries (Agilent Technologies, Santa Clara, CA) were generated and sequenced on a MiSeq NGS platform (Illumina, San Diego, CA) as previously described. ${ }^{4}$ Targeted enrichment included 258 known monogenic inherited retinal degeneration genes. ${ }^{3,4}$ For whole-exome sequencing (WES), targeted enrichment capture libraries were generated using the SureSelect Human All Exon targeted enrichment kit (V4+UTR, Agilent Technologies) and sequenced using a HiSeq 2000 NGS instrument (Illumina) as described previously. ${ }^{13}$ The NGS data were analyzed as previously described, ${ }^{4,13}$ with annotations taken from dbSNP (http://www.ncbi.nlm.nih.gov/ $\mathrm{SNP} /$ ), the Exome Aggregation Consortium (ExAC), the 1000 Genomes Project, UK10K project data (http://www.uk10k. org/), Genomic Evolutionary Rate Profiling, SIFT, PolyPhen2, and retinal expression. ${ }^{14}$ Rare variants were selected based on the minor allele frequency in public databases of less than $0.5 \%$.

Mutations were annotated based on the following transcripts: CNNM4 (NM_020184), DMD (NM_004007), EYS (NM_001142800), GPM6B (NM_001001995), KRT84 (NM_033045), MMGT1 (NM_173470), PDE6C (NM_006204), PNPLA4 (NM_004650), SNRNP200 (NM_014014), STARD9 (NM_020759), and ZNF449 (NM_152695).

\section{CNV analysis}

Genomic DNA (gDNA) samples from probands and family members were analyzed with dense whole-genome SNP microarrays (Human Omni 2.5 or Human Omni 5.0, Illumina) according to the manufacturer's instructions. The hybridized SNP arrays were analyzed using an array reader (iScan array scanner, Illumina) and the SNP calls were made with the genotyping module of the data analysis software (GenomeStudio, Illumina). A custom SNP genotyping report containing SNP chromosome coordinates, genotyping calls, $\log \mathrm{R}$ ratios, and B-allele frequencies was generated for each patient, followed by analysis using the CNV Workshop Suite. ${ }^{15}$

A genome-wide CGH array enriched with high-density probes against the IRD disease genes was designed with the eArray online tool $(4 \times 180 \mathrm{~K}$ microarray, Agilent Technologies $)$ as described previously. The high probe density enables detection of single exon deletions if more than one probe targets the exon. Samples were prepared using sex-matched control gDNA provided with the kit according to standard methods (SureTag Complete DNA Labeling Kit, Agilent Technologies). CGH analysis was performed using CytoGenomics software (Agilent Technologies).

\section{Quantitative real-time PCR}

Deletions were validated and fine-mapped using quantitative real-time PCR (qRT-PCR) on gDNA with primers specific to sequences flanking the presumed deletion breakpoint and normalized to two reference genes: ZNF80 and GPR15 (Supplementary Table S1 online). For each qRT-PCR reaction, $5 \mathrm{ng}$ of gDNA, $200 \mathrm{nmol} / \mathrm{l}$ of each primer and $10 \mu \mathrm{l}$ of Fast SYBR Green Master Mix (Life Technologies, Grand Island, NY) were used. The amplification was performed in a qPCR system (Stratagene Mx3000P, Agilent Technologies) using the standard thermocycling program: $95^{\circ} \mathrm{C}$ for $3 \mathrm{~min}, 40$ cycles of $95^{\circ} \mathrm{C}$ for $20 \mathrm{~s}$, and $60^{\circ} \mathrm{C}$ for $1 \mathrm{~min}$ followed by a melting curve. Each sample was assayed in triplicate, with two reference genes at each run (ZNF80 and GPR15). The standard deviation reflecting normalization to each of the reference genes was calculated and presented as the error bars.

\section{PCR and Sanger sequencing}

Validation of deletion breakpoints in families OGI-086 and OGI-036 was performed by PCR amplification using Herculase II fusion DNA Polymerase (Agilent Technologies) with the 
followingprimers:5'-GACAGAGAGAAACTGGTCTC-3'(OGI086-forward), 5'-CTTGTAGGTCAGGTGACTAG-3' (OGI086-reverse), 5'-TAATTACAAAGGATTTGCAGGGAACAA G-3' (OGI-036-forward), and 5'-CAGACGCAGTACGCAAA GAT-3' (OGI-036-reverse). Amplification conditions were as follows: $92{ }^{\circ} \mathrm{C}$ for $2 \mathrm{~min}$; 36 cycles of $92{ }^{\circ} \mathrm{C}$ for $20 \mathrm{~s}, 62^{\circ} \mathrm{C}$ for $30 \mathrm{~s}$, and $68^{\circ} \mathrm{C}$ for $1 \mathrm{~min}$ (OGI-086) and $92{ }^{\circ} \mathrm{C}$ for $2 \mathrm{~min}$; 36 cycles of $92{ }^{\circ} \mathrm{C}$ for $20 \mathrm{~s}, 58{ }^{\circ} \mathrm{C}$ for $30 \mathrm{~s}$, and $68{ }^{\circ} \mathrm{C}$ for $6 \mathrm{~min}$ (OGI-036). Sanger sequencing was performed after PCR cleanup (ExoSap-IT, Affymetrix, Santa Clara, CA, USA) and sequenced (BigDye Terminator v3.1, ABI 3730xl, Life Technologies) using the PCR primers.

\section{Generating NAHR prone regions}

A segmental duplication track was obtained from the USCS genome browser (http://genome.ucsc.edu/) using the GRCh37/ hg19 genome assembly. The regions were filtered to contain repeat pairs according to the following criteria: $>10-\mathrm{kb}$-long regions, $>95 \%$ identity, with intervening sequence between $50 \mathrm{~kb}$ and $10 \mathrm{Mb}$, and not spanning the centromere. Direct and inverted repeats are included. The overlapping regions were subsequently merged to obtain 156 regions spanning $\approx 300 \mathrm{Mb}$ of genomic sequence (Supplementary Table S2 online). Similar methodology was applied by Liu and colleagues. ${ }^{12}$ IRD genes were superimposed onto the NAHR regions (Figure 4) using NCBI Genome Decoration Page (http://www.ncbi.nlm. nih.gov/genome/tools/gdp)

\section{RESULTS}

Twenty-six families previously unsolved with WES and two families with apparently homozygous mutations in known IRD genes were studied for deletions with a dense whole-genome SNP array (Illumina Human Omni 2.5/5.0) and/or a CGH array. Using these techniques, we detected large deletions in IRD genes in five families (Table 1). These CNVs were further validated using PCR and quantitative real-time qRT-PCR.

Patient OGI-046-116 presented with retinitis pigmentosa (RP) at age 9 years. Fundus examination showed early bone spicule pigmentation in the retinal peripheries of both eyes. The patient had constricted visual fields and elevated dark adaptation thresholds (Supplementary Table S3 online). OCT imaging showed thinning of the peripheral retina and cystic changes in the macula. The proband and his parents were studied with WES, which revealed a hemizygous missense variant (c.3133C>A, p.Pro1045Thr) in SNRNP200 (MIM 601664), a gene typically associated with a dominant form of RP. ${ }^{16}$ This variant was previously reported in a Chinese cohort of LCA patients; however, no details of phenotype, family segregation, or functional analysis of the variant were available. ${ }^{17}$ The p.Pro1045Thr change is rare (2/104,468 alleles in the ExAC database) and the Pro1045 residue is conserved throughout all vertebrates available in the UCSC genome browser (Supplementary Figure S1 online); therefore, this variant was considered to be likely pathogenic as a recessive allele. The p.Pro1045Thr change was heterozygous in the father (OGI-046-114) but absent in the mother, which suggested the possibility of a deletion of SNRNP200 in trans with the missense mutation.

Results from a dense SNP chip indicated a deletion of $1.4 \mathrm{Mb}$ (chr2: 96741757-98188430) in the proband. CGH array analysis and estimation of the homozygosity stretch identified by WES narrowed the interval to $1.1 \mathrm{Mb}$ (chr2:96755045-97823903) (Figure 1a). This region encompasses 20 genes, including SNRNP200 and CNNM4 (MIM 607805), a gene associated with a cone-rod dystrophy, and amelogenesis imperfecta, ${ }^{18,19}$ known as Jalili syndrome. Further qPCR validation for all family members indicated that this is a de novo deletion (Figure 1C). The absence of the deletion in the mother was further confirmed by four heterozygous SNPs in ADRA2B (rs34667759), ASTL (rs183967306 and chr2:g.96803404C>T), and TMEM127 (rs13022177), all within the deleted region in the proband. In addition, whole-genome SNP analysis of the proband and his mother confirmed the family relationship.

Interestingly, we found another family with a deletion of the same region (family OGI-023, Figure 1d). In this family, the proband presented with the combination of cone dystrophy and amelogenesis imperfecta, typical of Jalili syndrome, at age 11 years (Supplementary Table S3 online). ${ }^{18,19}$ Targeted genetic testing showed a homozygous nonsense mutation (c.480G $>$ A, p.Trp160*) in CNNM4. Segregation analysis revealed that although the subject's father carried the mutation (Figure 1d), his mother's sequence was wild-type at this location. To determine if this result was due to a maternally inherited deletion involving CNNM4, we analyzed the family using a dense SNP chip and a CGH array. These analyses identified a $\sim 1.1-\mathrm{Mb}$ multigenic deletion involving CNNM4 and SNRNP200 in both the proband and the mother, which was confirmed and finemapped by qPCR analysis (Figure 1e). The qPCR analyses demonstrated that the deletion in both families occurs between the GPAT2 and ANDRA2B genes on the $5 \square$ side and between $A N K R D 36$ and $Z A P 70$ on the $3 \rrbracket$ end. Unfortunately, we were not able to map the exact breakpoints in this family due to highly repetitive regions near the centromere. These highly repetitive regions flanking the deletion were also informative about the possible mechanism of this $\mathrm{CNV}$ because recurrent genomic rearrangements often occur through NAHR, between long highly homologous sequences on the same chromosome. ${ }^{12}$ We found that the deleted region is flanked by $\approx 16-\mathrm{kb}$ repeats bearing $98.5 \%$ sequence identity (chr2: 96598520-96614694 and chr2: 97820062-97836456).

Patient OGI-086-213 was diagnosed with RP at age 13 years. During his last visit at age 18 years, he was found to have bilateral constricted visual fields and reduced ERG responses, bone spicule pigmentation in the retinal periphery, and granularity in the macula (Supplementary Table S3 online). In addition, examination of the anterior chamber of the eye showed bilateral anterior polar cataracts. Similar cataracts were also present in the father, who did not have any symptoms of visual impairment and whose retina showed no clinical signs of degeneration on fundus examination. WES analysis showed rare variants in seven genes (Supplementary Table S4 online), none of which 
Table 1 Deletions detected in the IRD families

\begin{tabular}{|c|c|c|c|c|c|c|}
\hline \multirow[b]{2}{*}{ Family } & \multicolumn{3}{|c|}{ Deletion from SNP chip } & \multicolumn{2}{|l|}{ Final deletion } & \multirow[b]{2}{*}{ IRD Gene affected } \\
\hline & Position & Size & $\begin{array}{l}\text { No. } \\
\text { SNPs }\end{array}$ & Position & Size & \\
\hline OGI-046 & chr2: 96741757-98188430 & $1.4 \mathrm{Mb}$ & 558 & $\approx c h r 2: 96755045-97823903$ & $\approx 1.1 \mathrm{Mb}$ & SNRNP200, CNNM4 \\
\hline OGI-023 & chr2: 96688990-97765355 & $1.1 \mathrm{Mb}$ & $1,099^{a}$ & $\approx c h r 2: 96755045-97823903$ & $\approx 1.1 \mathrm{Mb}$ & SNRNP200, CNNM4 \\
\hline OGI-086 & chr19: 54604319-54632673 & $28.3 \mathrm{~kb}$ & 34 & chr19: 54602436-54636367 & $33,932 \mathrm{bp}$ & PRPF31 \\
\hline OGI-014 & chr6: 65604204-65655039 & $50.8 \mathrm{~kb}$ & 36 & chr6:(65659138-65658256)-(65602857-65602460) & $\approx 55.4-56.7 \mathrm{~kb}$ & EYS (ex15-18) \\
\hline
\end{tabular}

IRD, inherited retinal degeneration; SNP, single-nucleotide polymorphism.

aThis sample was analyzed using the Omni 5.0 SNP array.

was previously associated with IRD. CNV analysis performed using the SNP array revealed a heterozygous 28 -kb deletion on chromosome 19 in the proband and the father that affected four genes: OSCAR, NDUFA3, TFPT, and PRPF31 (Table 1). Mutations in PRPF31 are known to cause a dominant form of RP (MIM 600138), where the disease is caused by haploinsufficiency, and large deletions have been documented to be pathogenic. ${ }^{11}$ In addition, partial penetrance is a common feature in families with mutations in this gene, where the wild-type allele can have a disease-rescuing effect. ${ }^{20,21}$ Therefore, the unaffected status of the father was not surprising. The large deletion, which included PRPF31, was considered as the most likely cause of disease in the proband. This deletion was subsequently confirmed by qPCR and the breakpoints were PCR-amplified and Sanger-sequenced, which showed the deletion to be exactly 33,932 bp (chr19:54602436-54636367) (Figure 2).

Patient OGI-014-038 had had decreased vision and difficulty with night vision since age 15 years. He was diagnosed with RP at age 33 years (Supplementary Table S3 online, Figure 3a,b). The proband and his unaffected family members were genetically investigated with WES and with the dense SNP array. WES analysis revealed rare coding changes indicative of recessive disease in three genes: PNPLA4 (homozygous c.575C >T, p.Pro192Leu); KRT84 (heterozygous c.1327C >T, p.Arg443Trp and heterozygous c.364T>G, p.Phe122Val); and STARD9 (heterozygous c.5584_5589del, p.Ser1862_Thr1863del and heterozygous c.8803G>C, p.Glu2935Gln). None of these was previously associated with IRD. Additional rare coding changes in trans with noncoding variants in five genes were identified (Supplementary Table S4 online). Two heterozygous variants in known autosomal-recessive IRD genes were found: a paternally inherited mutation in EYS (c.9036delT, p.Leu3013Sfs ${ }^{\star} 6$ ) and a maternally inherited variant in PDE6C (c.413T $>$ C, p.Leu138Ser). These sequence alterations were not sufficient to explain the disease; however, the subsequent $\mathrm{CNV}$ analysis revealed a heterozygous maternally inherited deletion of approximately $51 \mathrm{~kb}$ in EYS encompassing exons 15-18 and leading to a frameshift (c.2260_2846del, p.Ser754Ifs ${ }^{\star} 3$ ). The above data suggest that EYS is the most likely cause of the disease in this patient. The EYS deletion was further mapped by qPCR, demonstrating that the deletion is between 55.4 and
$56.7 \mathrm{~kb}$ in size, where the $5 \rrbracket$ breakpoint is $2.5-3.4 \mathrm{~kb}$ upstream of exon 15 (chr6:65659138-65658256) and the $3 \otimes$ breakpoint is 9.2-9.6 kb downstream of exon 18 (chr6:65602857-65602460) (Figure 3c). Unfortunately, despite numerous attempts, we were not able to PCR-amplify across the breakpoints. This could be the result of a more complex genomic rearrangement, such as translocation.

Patient OGI-036-091 and his half-brother OGI-036336 presented with decreased visual acuity, reduced cone responses on full-field ERG testing, and decreased color vision (Supplementary Table S3 online; Supplementary Figure S2a online). Dark adaptation was within normal limits. Fundus examination showed pigment granularity in the macula and OCT imaging showed normal retinal architecture and thickness in the periphery with a diminished photoreceptor layer centrally, indicative of cone dystrophy. Family history was consistent with an X-linked inheritance pattern because the maternal grandfather was reported to have a retinal dystrophy (no clinical data available) (Supplementary Figure S2b online). The family was studied with WES to detect causative variants on chrX. Using the frequency criteria described in the Methods, we detected only four variants shared by the three affected males (OGI-036-091, 336, and 753), heterozygous in the obligate carrier (OGI-036-333), and absent in the father (OGI-036$334)$. These were three $3 \rrbracket$ UTR variants $\left(G P M 6 B\right.$ c. ${ }^{*} 525 \mathrm{~T}>\mathrm{C}$, ZNF449 c.*1661A $>\mathrm{G}$, and MMGT1 c.*2599T>C) and one intronic change ( $D M D \quad c .7173+13 \mathrm{~A}>\mathrm{G})$ (Supplementary Table S4 online). Because none of these variants seemed likely pathogenic, we performed SNP array analysis to detect deletions. Using this technique, we detected a $15.6-\mathrm{kb}$ deletion affecting the promoter region and exons 1-4 of OPN1LW (Table 1; Supplementary Figure S2c online), a gene known to be implicated in blue cone monochromacy. ${ }^{22}$ PCR amplification of selected regions (Supplementary Figure S2d online) as well as amplification and Sanger sequencing across the breakpoints (Supplementary Figure S2e online) revealed a much larger deletion of 52,664bp, which affects the entire OPN1LW gene with its promoter and the first exon of the adjacent OPN1MW (Supplementary Figure S2f online). In this family, the genetic diagnosis helped to refine the clinical diagnosis, which is likely blue cone monochromacy. 
a

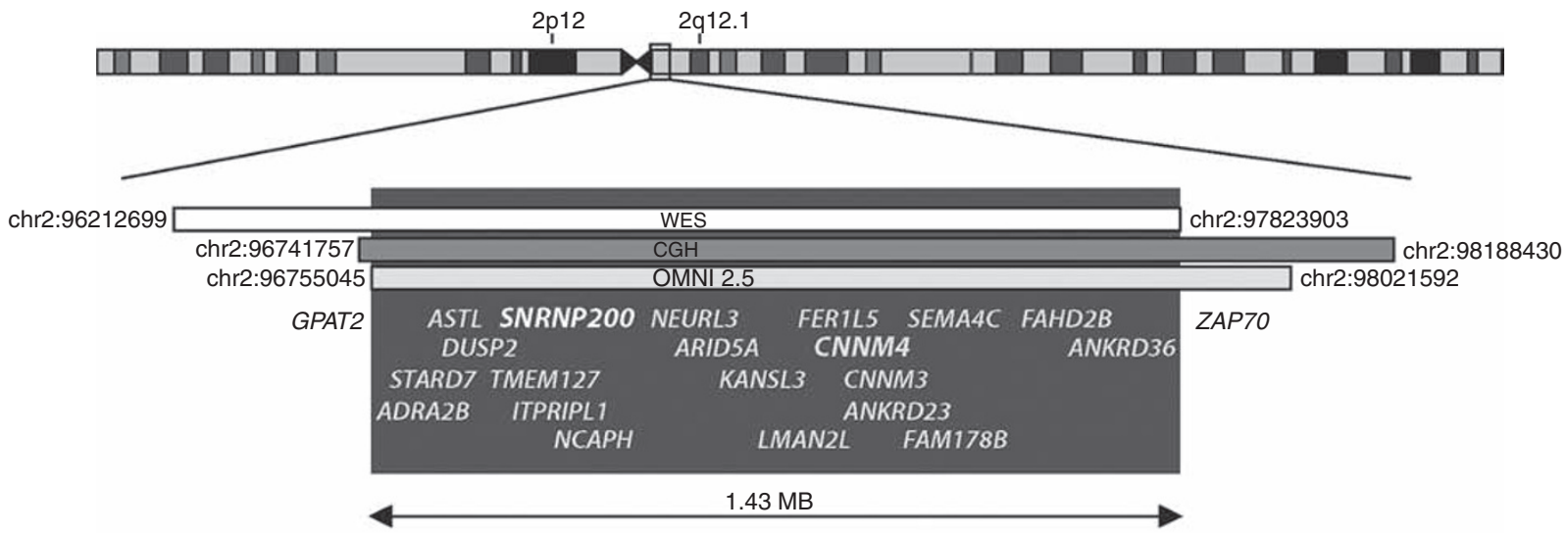

b

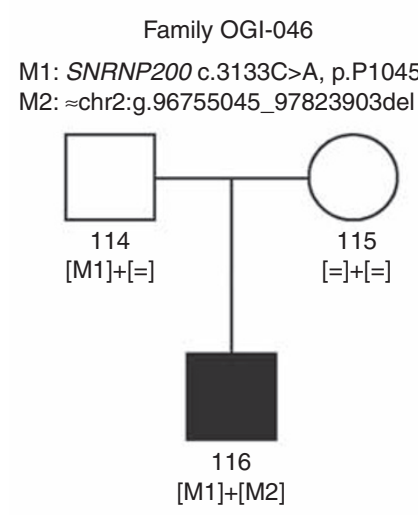

d

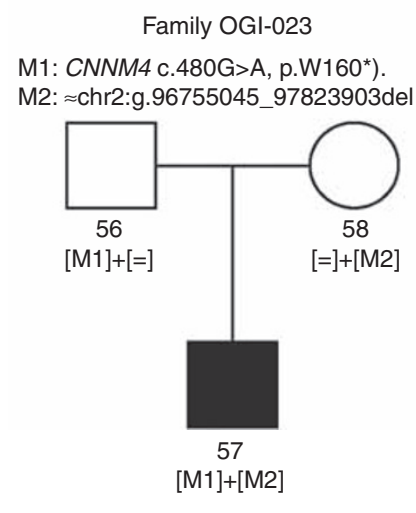

C

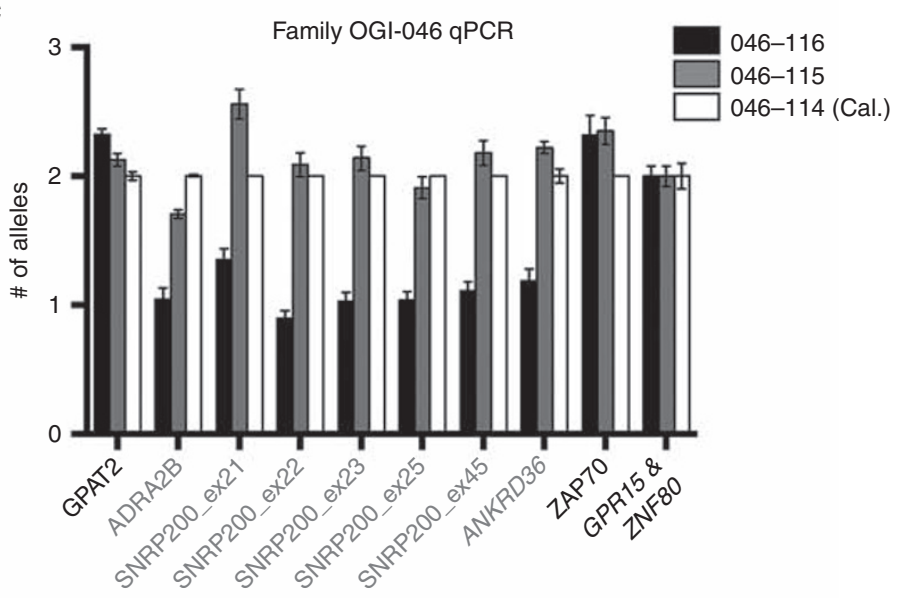

$\mathbf{e}$

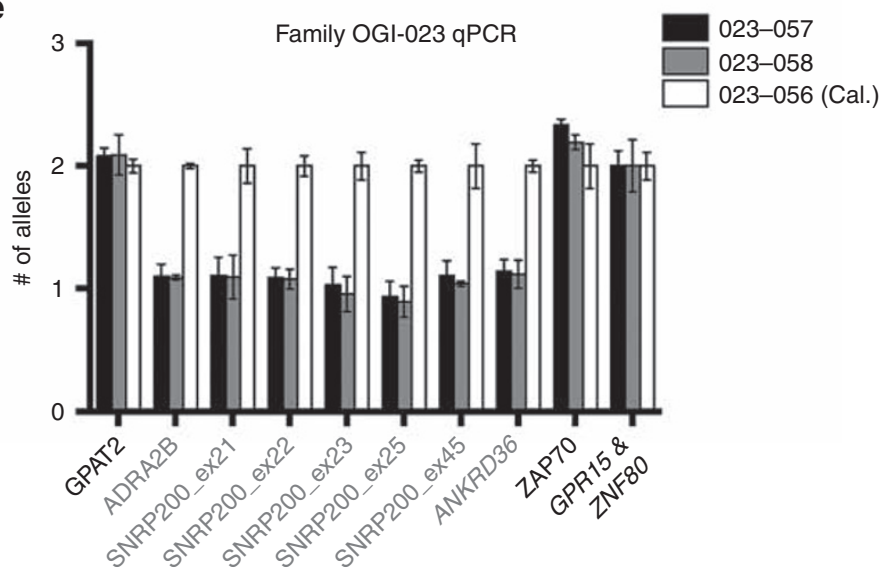

Figure 1 Deletion mapping in families OGI-046 and OGI-023. (a) A heterozygous deletion of 20 genes on chr2q12.1 found in family OGI-046 by CGH and SNP arrays and validated by SNPs found in whole-exome sequencing. (b) Pedigree of family OGI-046 with mutant allele segregation indicated. (c) Fine mapping of the deletion by quantitative polymerase chain reaction (qPCR) in family OGI-046. (d) Pedigree of family OGI-023 with mutant allele segregation indicated. (e) Fine mapping of the deletion by qPCR in family OGI-023. Each primer pair in the qPCR experiments was normalized to two reference genes, GPR15 and ZNF80 (error bars represent the standard deviation between these results).

\section{DISCUSSION}

The results reported here indicate that CNVs contribute significantly to the genetic causality of IRDs. Of the 28 patients whose genetic cause of disease was not identified by panel-based NGS testing or WES, likely disease-causing deletions were identified in five (or 18\%) cases. Four of the patients with deletions came from an original WES cohort of 56 patients; therefore, we estimate that $\approx 7 \%$ of IRD patients carry large deletions in known IRD genes. This result has important implications for genetic diagnostic testing of patients with IRDs, as well as for studies seeking to identify novel IRD disease genes. The finding of the same deletion in two families with distinct causes of disease 
Family OGI-086

M: chr19:54602436_54636367del

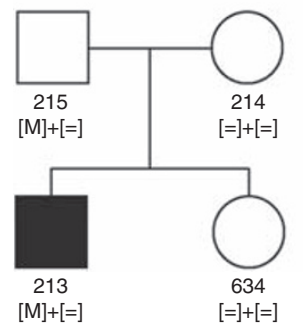

C

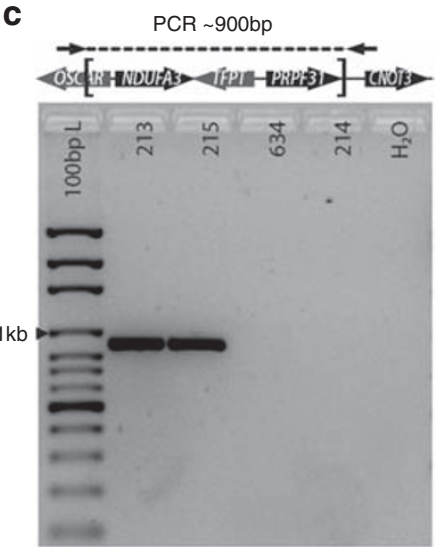

b
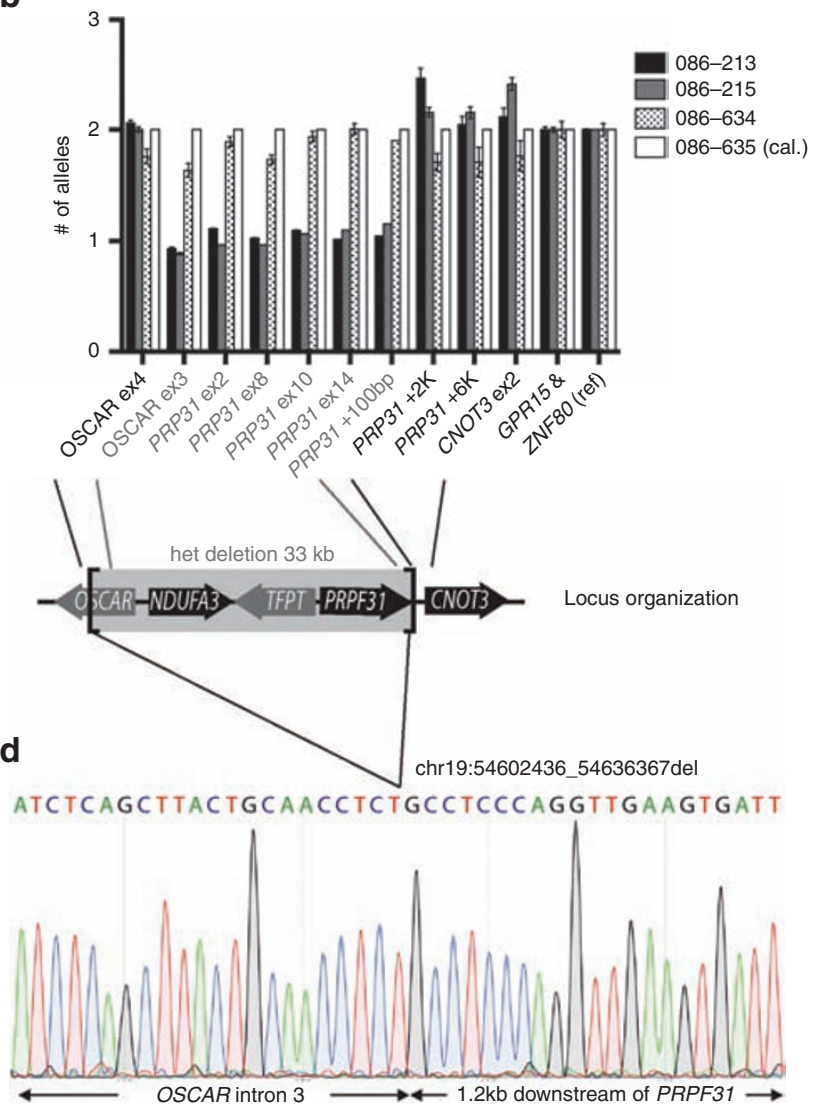

Figure 2 Deletion mapping in family OGI-086. (a) Pedigree of family OGI-086 with mutant allele segregation. (b) Fine mapping of the deletion by qPCR with deleted genes indicated (error bars represent standard deviation between two normalization genes, GPR15 and ZNF80). (c) Polymerase chain reaction amplification across the breakpoints. (d) Sanger sequencing electropherogram showing the precise position of the deletion.

a

Family OGI-014

M1: EYS c.9036delT (p.Leu3013Sts*6) M2: $E Y S \approx 55 \mathrm{~kb}$ deletion (ex15-18)
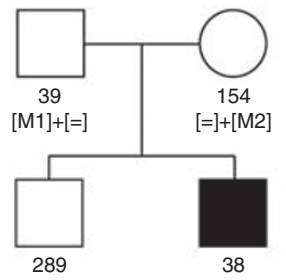

$[\mathrm{M} 1]+[=]$

b

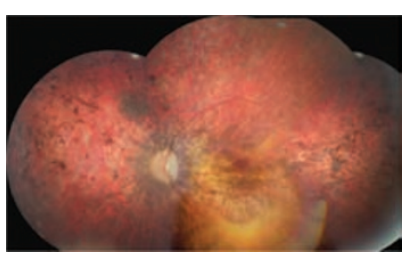

C

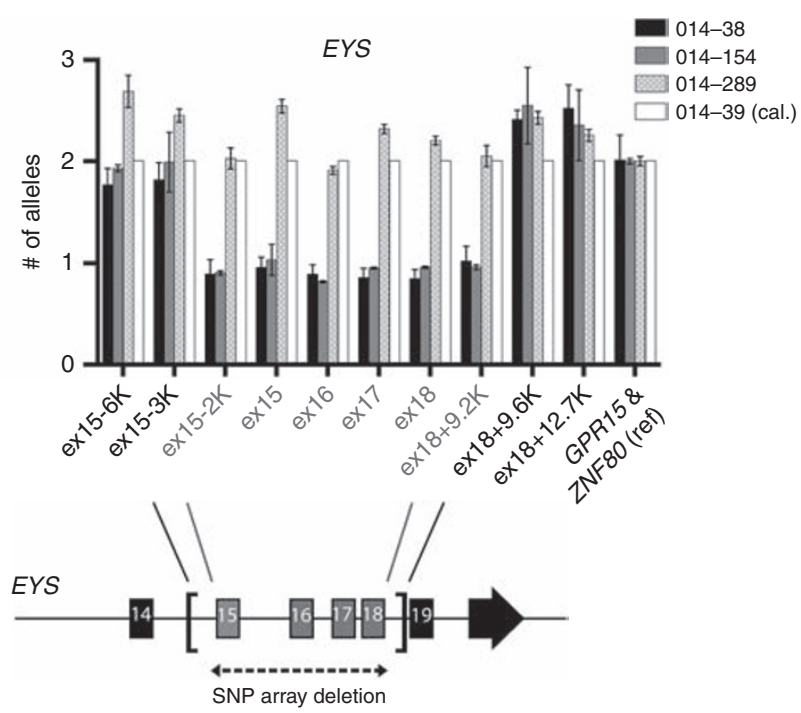

Figure 3 Deletion mapping in family OGI-014. (a) Pedigree of family OGI-014 with mutant allele segregation. (b) Composite fundus photograph of the left eye of patient OGI-014-038. (c) Fine mapping of the deletion by quantitative polymerase chain reaction with four deleted exons of $E Y S$ indicated (error bars represent standard deviation between two normalization genes, GPR15 and ZNF80). 
suggests that previously identified mechanisms that create SVs in the genome, such as NAHR, can create pathogenic alleles in IRD genes. ${ }^{12,23}$ This suggests that methods capable of detecting more complex forms of genomic rearrangement, such as WGS or single-molecule long-read sequencing, will help identify novel genetic causes of IRD. ${ }^{8,24,25} \mathrm{SV}$ s can also be detected by other techniques such as fluorescence in situ hybridization (FISH); however, this technique is more useful to test a candidate genomic rearrangement or validate results from other methods rather than unbiased detection of genome-wide SVs. ${ }^{26}$

Patients from two unrelated families carried essentially the same deletion, which spanned two known IRD disease genes: SNRNP200 (ref. ${ }^{16}$ ) and CNNM4 (refs. ${ }^{18,19}$ ). The deletion in patient OGI-023-057 was maternally inherited; in addition, he carried a paternally inherited stop mutation in CNNM4, which correlated with his phenotype of cone dysfunction and amelogenesis imperfecta as part of Jalili syndrome and the recessive inheritance of CNNM4-associated retinal degeneration. ${ }^{18,19}$ The large deletion in patient OGI-046-116 occurred de novo and was accompanied by a likely pathogenic missense change in SNRNP200 (p.Pro1045Thr) inherited from his father. The proband showed typical signs of nonsyndromic rod-cone dystrophy (Supplementary Table S3 online). ${ }^{1,2}$ This genotype is unusual because SNRNP200 was previously associated with dominantly inherited retinal degeneration. ${ }^{16,27}$ There is only one report of recessive disease due to mutations in SNRNP200. ${ }^{17}$ SNRNP200 codes for an RNA helicase hBRR2, a component of the pre-mRNA spliceosome. ${ }^{28}$ Mutations in other splicing genes (PRPF3, PRPF4, PRPF6, PRPF8, PRPF31, RP9) were also associated with dominant $\mathrm{RP},{ }^{29-34}$ in which the disease mechanism in patients with PRPF31 mutations was shown to be haploinsufficiency. ${ }^{20,21}$ Therefore, originally, we hypothesized that the disease in patient OGI-046-116 was due to the haploinsufficiency of SNRNP200 and that the p.Pro1045Thr change had no functional effect, even though the amino acid residue was highly conserved (Supplementary Figure S1 online). However, because we discovered another family with a large deletion encompassing SNRNP200, where the deletion carrier is unaffected (OGI-023-058), we concluded that missing one copy of SNRNP200 is not sufficient to cause the disease. We believe that the p.Pro1045Thr mutation probably creates a hypomorphic allele, and the combination of hemizygosity for SNRNP200 with the p.Pro1045Thr allele is compatible with life but insufficient to maintain a healthy retina. Alternatively, the disease mechanism in SNRNP200-associated IRD is due to haploinsufficiency and the partial penetrance is due to other genetic factors, such as SNRNP200 expression level from the wild-type allele, as was demonstrated for PRPF31 (refs. ${ }^{20,21}$ ). To test these hypotheses, however, a larger cohort of patients with SNRNP200 deletions is needed, and so far the only reported mutations were missense changes and one splice-site variant. ${ }^{27}$

Another unusual finding is that both families OGI-023 and OGI-046 carried the same deletion that occurred de novo in patient OGI-046-116. Such recurrent genomic rearrangements are thought to result from NAHR, where the rearranged sequence is flanked by paralogous repeat sequences. ${ }^{12,35}$ The deleted interval of approximately $1.1 \mathrm{Mb}$ is flanked by $\approx 16-\mathrm{kb}$ repeats bearing $98.5 \%$ sequence identity. This region was also previously predicted to be prone to NAHR. ${ }^{12}$ Therefore, even though we are the first to report this $1.1-\mathrm{Mb}$ deletion encompassing SNRNP200 and CNNM4 associated with inherited retinal degenerations, we expect that this is a more common event. Deletion of this large region also carries additional implications because other deleted genes may lead to phenotypes unrelated to IRDs; for example, heterozygous loss-of-function mutations in TMEM127 have been associated with susceptibility to pheochromocytoma. ${ }^{36}$ None of the subjects from families OGI023 and OGI-046 with a heterozygous deletion in TMEM127 was reported to have pheochromocytoma or any other type of cancer.

Encouraged by this example, we hypothesized that other IRD genes may fall into the NAHR-prone regions and have recurrent CNVs. We therefore constructed a genome-wide map of NAHR regions and superimposed known IRD genes implicated in isolated and syndromic disease (Figure 4). We found that 35 IRD genes are likely to be affected by NAHR, 13 of which were never associated with a CNV before, 9 had large indels affecting only some exons of the gene, and the remaining 13 had CNVs

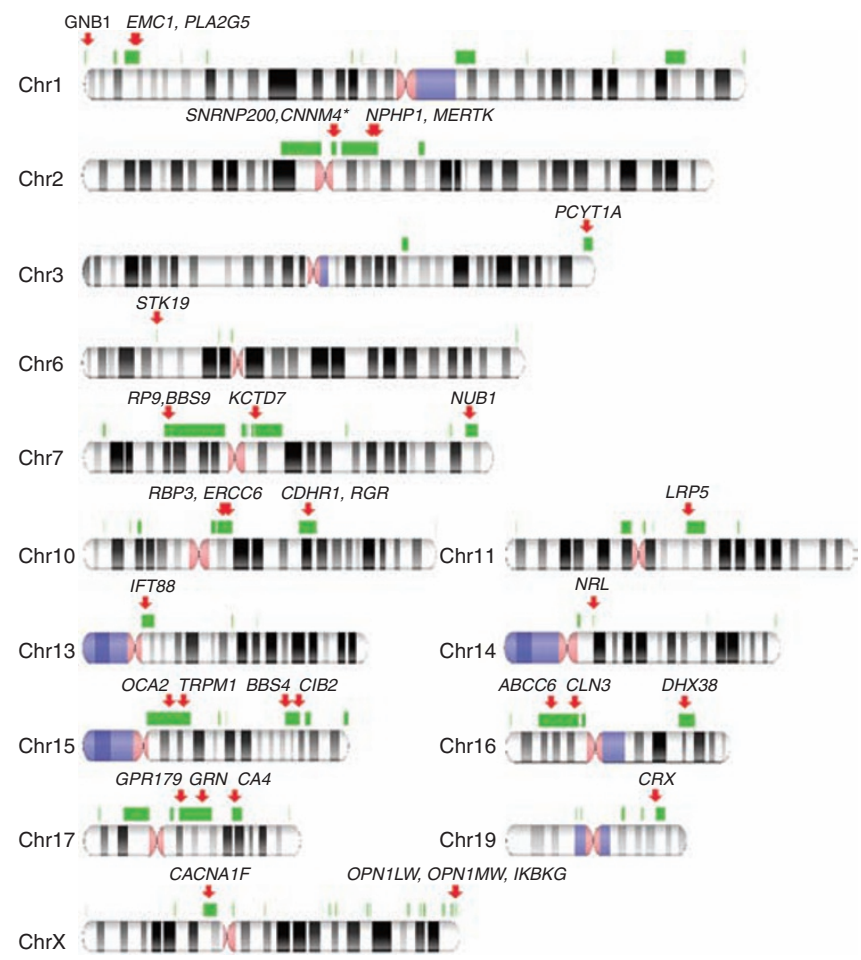

Figure 4 Nonallelic homologous recombination prone regions and inherited retinal degeneration (IRD) genes. Nonallelic homologous recombination (NAHR)-prone regions (green bars) were computationally predicted using the following criteria: $>10-\mathrm{kb}-$ long regions of $>95 \%$ identity, with intervening sequence between $50 \mathrm{~kb}$ and $10 \mathrm{Mb}$ and not spanning the centromere. Direct and inverted repeats are included. IRD genes overlapping the NAHR-prone regions are indicated by red arrows. Only the chromosomes where NAHR-prone regions and IRD genes overlap are presented. The full genome-wide map of NAHR-prone regions is presented in Supplementary Table S2 online. *IRD whole-gene CNVs reported in this article for the first time. 
affecting the entire gene reported (Figure 4; Supplementary Table S2 online) ${ }^{27}$ Among the genes likely affected by NAHR were OPN1LW and OPN1MW; the deletion was associated with blue cone monochromacy in this study (family OGI036). Other researchers have also reported deletions in this region, ${ }^{37,38}$ which validates our predictions of regions prone to recombination. We believe that identifying such recombination hotspots will facilitate searching for the missing genetic causality in IRD patients and perhaps in patients with other Mendelian disorders.

The disease in the remaining two families was due to deletions in PRPF31 and EYS. Even though new deleted regions were found in this study, structural variations in these genes were reported previously. ${ }^{11,39,40}$ Deletions in PRPF31 and EYS were also found to be major contributors to IRD pathology by other researchers, accounting for $2.5 \%$ of adRP and $4 \%$ of previously unsolved arRP cases, respectively. ${ }^{11,39}$ Because these genes do not lie in proximity of the NAHR regions, other mechanisms of structural variations are likely implicated. ${ }^{35}$ Before ascertaining that the disease in proband OGI-014-038 is due to disruption of EYS, there were three potential candidates for new genetic types of IRD. Variants in these three genes (PNPLA4, KRT84, and STARD9) were rare, affecting conserved residues, and the genes were expressed in the retina (Supplementary Table $\mathbf{S 4}$ online). It therefore seems imperative that CNVs in the known disease genes be investigated before new gene-disease associations are reported.

In summary, we detected deletions that contribute to the disease in 5 out of $28 \mathrm{IRD}$ patients. The high rate of CNVs $(\approx 18 \%)$ in the families who were not previously solved by the targeted NGS approaches indicates that these types of mutations are the frequent cause of the missing inheritance in IRDs and that CNV detection should be included in genetic diagnostic testing. We expect that the integrated approach of the targeted NGS and CNV mapping could provide a genetic diagnosis for up to $67 \%$ of IRD cases (60\% by $\mathrm{NGS}^{4-7}$ and an additional $7 \%$ from CNV mapping). The techniques used in the present study (SNP and CGH arrays) can only detect large deletions or duplications, which is inherent to these techniques that rely on the color or strength of the fluorescence signal after hybridization of the gDNA to specific probes complementary to the target regions of the genome. Smaller CNVs, which are not targeted by the genome-wide probes in the arrays, as well as translocations, inversions, or other complex structural rearrangements, which will not alter the intensity of the fluorescence signal, cannot be detected by SNP and CGH arrays. Consequently, it is possible that SVs in known IRD genes are more important contributors to disease than detected in this study. Other researchers proposed using the NGS read-depth to infer CNVs, which also increased the genetic diagnostic rate of IRDs. ${ }^{40}$ The read-depth analysis of NGS reads has similar limitations; therefore, whole-genome sequencing may be necessary to map certain SVs, as shown previously. ${ }^{8}$

\section{SUPPLEMENTARY MATERIAL}

Supplementary material is linked to the online version of the paper at http://www.nature.com/gim

\section{ACKNOWLEDGMENTS}

This work was supported by grants from the National Eye Institute (EY012910 (EAP) and P30EY014104 (MEEl core support)), the Foundation Fighting Blindness (USA, EAP), and the Fleming Family Foundation (KMB). BPL is a Senior Clinical Investigator of the Research Foundation Flanders (FWO), Belgium. The authors thank the patients and their family members for their participation in this study and the Ocular Genomics Core members for their experimental assistance. The authors also thank the Exome Aggregation Consortium and the groups that provided exome variant data for comparison. A full list of contributing groups can be found at http://exac.broadinstitute. org/about.

\section{DISCLOSURE}

The authors declare no conflict of interest.

\section{REFERENCES}

1. Berger W, Kloeckener-Gruissem B, Neidhardt J. The molecular basis of human retinal and vitreoretinal diseases. Prog Retin Eye Res 2010;29:335-375.

2. Hartong DT, Berson EL, Dryja TP. Retinitis pigmentosa. Lancet 2006;368:1795-1809.

3. Retinal Information Network (RetNet). https://sph.uth.edu/retnet/sum-dis. htm\#A-genes. Accessed May 2016.

4. Consugar MB, Navarro-Gomez D, Place EM, et al. Panel-based genetic diagnostic testing for inherited eye diseases is highly accurate and reproducible, and more sensitive for variant detection, than exome sequencing. Genet Med 2015;17:253-261.

5. Audo I, Bujakowska KM, Léveillard T, et al. Development and application of a next-generation-sequencing (NGS) approach to detect known and novel gene defects underlying retinal diseases. Orphanet J Rare Dis 2012;7:8.

6. Neveling K, Collin RWJ, Gilissen C, et al. Next generation genetic testing for retinitis pigmentosa. Hum Mutat 2013;34:1181-1181.

7. Huang X-F, Huang F, Wu K-C, et al. Genotype-phenotype correlation and mutation spectrum in a large cohort of patients with inherited retinal dystrophy revealed by next-generation sequencing. Genet Med 2015;17:271-278.

8. Nishiguchi KM, Tearle RG, Liu YP, et al. Whole genome sequencing in patients with retinitis pigmentosa reveals pathogenic DNA structural changes and NEK2 as a new disease gene. Proc Natl Acad Sci USA 2013;110:16139-16144.

9. Bitner-Glindzicz M, Lindley KJ, Rutland $P$, et al. A recessive contiguous gene deletion causing infantile hyperinsulinism, enteropathy and deafness identifies the Usher type 1C gene. Nat Genet 2000;26:56-60.

10. den Hollander Al, Koenekoop RK, Yzer S, et al. Mutations in the CEP290 (NPHP6) gene are a frequent cause of Leber congenital amaurosis. Am J Hum Genet 2006;79:556-561.

11. Sullivan LS, Bowne SJ, Seaman CR, et al. Genomic rearrangements of the PRPF31 gene account for $2.5 \%$ of autosomal dominant retinitis pigmentosa. Invest Ophthalmol Vis Sci 2006;47:4579-4588.

12. Liu P, Carvalho CM, Hastings PJ, Lupski JR. Mechanisms for recurrent and complex human genomic rearrangements. Curr Opin Genet Dev 2012;22: 211-220.

13. Bujakowska KM, Zhang Q, Siemiatkowska AM, et al. Mutations in IFT172 cause isolated retinal degeneration and Bardet-Biedl syndrome. Hum Mol Genet 2015;24:230-242.

14. Farkas MH, Grant GR, White JA, Sousa ME, Consugar MB, Pierce EA. Transcriptome analyses of the human retina identify unprecedented transcript diversity and $3.5 \mathrm{Mb}$ of novel transcribed sequence via significant alternative splicing and novel genes. BMC Genomics 2013;14:486.

15. Gai X, Perin JC, Murphy K, et al. CNV Workshop: an integrated platform for high-throughput copy number variation discovery and clinical diagnostics. BMC Bioinformatics 2010;11:74.

16. Zhao C, Bellur DL, Lu S, et al. Autosomal-dominant retinitis pigmentosa caused by a mutation in SNRNP200, a gene required for unwinding of U4/U6 snRNAs. Am J Hum Genet 2009;85:617-627.

17. Wang $X$, Wang H, Sun V, et al. Comprehensive molecular diagnosis of 179 Leber congenital amaurosis and juvenile retinitis pigmentosa patients by targeted next generation sequencing. J Med Genet 2013;50:674-688.

18. Polok $B$, Escher $P, A m b r e s i n ~ A$, et al. Mutations in CNNM4 cause recessive cone-rod dystrophy with amelogenesis imperfecta. Am J Hum Genet 2009;84:259-265. 
19. Parry DA, Mighell AJ, El-Sayed W, et al. Mutations in CNNM4 cause Jalili syndrome, consisting of autosomal-recessive cone-rod dystrophy and amelogenesis imperfecta. Am J Hum Genet 2009;84:266-273.

20. Rivolta C, McGee TL, Rio Frio T, Jensen RV, Berson EL, Dryja TP. Variation in retinitis pigmentosa-11 (PRPF31 or RP11) gene expression between symptomatic and asymptomatic patients with dominant RP11 mutations. Hum Mutat 2006;27:644-653.

21. Rose AM, Shah AZ, Venturini G, et al. Transcriptional regulation of PRPF31 gene expression by MSR1 repeat elements causes incomplete penetrance in retinitis pigmentosa. Sci Rep 2016;6:19450.

22. Nathans J, Merbs SL, Sung CH, Weitz CJ, Wang Y. Molecular genetics of human visual pigments. Annu Rev Genet 1992;26:403-424.

23. Lindstrand A, Davis EE, Carvalho CM, et al. Recurrent CNVs and SNVs at the NPHP1 locus contribute pathogenic alleles to Bardet-Biedl syndrome. Am J Hum Genet 2014;94:745-754.

24. Fromer M, Moran JL, Chambert K, et al. Discovery and statistical genotyping of copy-number variation from whole-exome sequencing depth. Am J Hum Genet 2012;91:597-607.

25. Huang DW, Raley C, Jiang MK, et al. Towards better precision medicine: PacBio single-molecule long reads resolve the interpretation of HIV drug resistant mutation profiles at explicit Quasispecies (Haplotype) level. J Data Mining Genomics Proteomics 2016;7:182

26. Bejjani BA, Shaffer LG. Clinical utility of contemporary molecular cytogenetics. Annu Rev Genomics Hum Genet 2008;9:71-86.

27. Human Gene Mutation Database (HGMD). http://www.hgmd.cf.ac.uk/ac/ index.php. Accessed May, 2016.

28. Liu S, Rauhut R, Vornlocher HP, Lührmann R. The network of protein-protein interactions within the human U4/U6.U5 tri-snRNP. RNA 2006:12:1418-1430.

29. Vithana EN, Abu-Safieh L, Allen MJ, et al. A human homolog of yeast pre-mRNA splicing gene, PRP31, underlies autosomal dominant retinitis pigmentosa on chromosome 19q13.4 (RP11). Mol Cell 2001;8:375-381.
30. Chakarova CF, Hims MM, Bolz H, et al. Mutations in HPRP3, a third member of pre-mRNA splicing factor genes, implicated in autosomal dominant retinitis pigmentosa. Hum Mol Genet 2002;11:87-92.

31. Chen $X$, Liu $Y$, Sheng $X$, et al. PRPF4 mutations cause autosomal dominant retinitis pigmentosa. Hum Mol Genet 2014;23:2926-2939.

32. Tanackovic G, Ransijn A, Ayuso C, Harper S, Berson EL, Rivolta C. A missense mutation in PRPF6 causes impairment of pre-mRNA splicing and autosomaldominant retinitis pigmentosa. Am J Hum Genet 2011;88:643-649.

33. McKie AB, McHale JC, Keen TJ, et al. Mutations in the pre-mRNA splicing factor gene PRPC8 in autosomal dominant retinitis pigmentosa (RP13). Hum Mol Genet 2001;10:1555-1562.

34. Keen TJ, Hims MM, McKie AB, et al. Mutations in a protein target of the Pim-1 kinase associated with the RP9 form of autosomal dominant retinitis pigmentosa. Eur J Hum Genet 2002;10:245-249.

35. Malhotra D, Sebat J. CNVs: harbingers of a rare variant revolution in psychiatric genetics. Cell 2012;148:1223-1241.

36. Qin $Y$, Yao L, King EE, et al. Germline mutations in TMEM127 confer susceptibility to pheochromocytoma. Nat Genet 2010;42:229-233.

37. Carroll J, Rossi EA, Porter J, et al. Deletion of the X-linked opsin gene array locus control region (LCR) results in disruption of the cone mosaic. Vision Res 2010;50:1989-1999.

38. Ayyagari $R$, Kakuk LE, Coats $C L$, et al. Bilateral macular atrophy in blue cone monochromacy (BCM) with loss of the locus control region (LCR) and part of the red pigment gene. Mol Vis 1999;5:13

39. Pieras JI, Barragán I, Borrego S, et al. Copy-number variations in EYS: a significant event in the appearance of arRP. Invest Ophthalmol Vis Sci 2011;52:5625-5631.

40. Eisenberger $\mathrm{T}$, Neuhaus $\mathrm{C}$, Khan $\mathrm{AO}$, et al. Increasing the yield in targeted next-generation sequencing by implicating CNV analysis, non-coding exons and the overall variant load: the example of retinal dystrophies. PLoS One 2013;8:e78496. 